\title{
Student understanding of the measurable effects of relative phases in superposition states
}

\author{
Tong Wan ${ }^{1}$, Paul J. Emigh ${ }^{2}$, and Peter S. Shaffer ${ }^{1}$ \\ ${ }^{1}$ Department of Physics, University of Washington, Seattle, WA 98195 \\ ${ }^{2}$ Department of Physics, Oregon State University, Corvallis, OR 97331
}

\begin{abstract}
Quantum states have complex probability amplitudes that are sometimes represented by positive real numbers multiplied by complex exponentials. Although the overall phase of a superposition state does not affect the probabilities, the relative phases between the component basis states can have measurable effects. A thorough grasp of relative phase is needed for students to understand various key ideas in quantum mechanics, including quantum interference and time dependence. We present preliminary results from an investigation into student understanding of the measurable effects of relative phases that was conducted in sophomore- and junior-level quantum mechanics courses at the University of Washington (UW). The findings suggest that many students do not recognize that relative phases have measureable effects and tend to overlook the important role that complex numbers play in quantum mechanics.
\end{abstract}

\section{INTRODUCTION}

Prior research has demonstrated some of the difficulties that students have in reasoning about superposition states in quantum mechanics. At the most basic level, there is evidence that many students are not able to distinguish between superposition states and mixed states [1]. In this paper, we focus on an important aspect of student understanding of superposition states: relative phases and their measurable effects.

Complex numbers are used in both classical and quantum physics. In classical mechanics, they are used primarily to simplify computation. However, they are essential to quantum mechanics. Both the Schrödinger equation and the probability amplitudes are complex. Although probability amplitudes themselves are not measurable quantities, the relative phase(s) between probability amplitudes affect real-world measurements. For example, quantum interference phenomena can be explained by the dependence of the probabilities on relative phase, which illustrates the importance of complex numbers. When a superposition state evolves in time, the relative phase also changes in time, resulting in timedependent probabilities. Thus, an understanding of the measurable effects of relative phases is a key step to grasping more challenging ideas in quantum mechanics.

There is relatively little research on student understanding of the impact on measurements of the phases in superposition states [2,3]. Close et al. have found that nested phasor diagrams can be used productively to represent two complex coefficients for spin-1/2 states in order to help visualize the relative phase between two basis states [3]. Others have shown that difficulty with complex numbers can hinder student fluency with the mathematical formalism in quantum mechanics [4-6].

The purpose of this study is to investigate whether or not students recognize that (1) relative phases in superposition states have measurable effects and (2) complex numbers are essential in quantum theory. In this article, we discuss open-ended questions used to probe students' conceptual understanding of how relative phases impact measurements. In some questions, students are given two quantum states that differ only by a relative phase and asked whether or not those states are experimentally distinguishable. We also analyze performance on other questions that ask students to compare probabilities for a specific observable.

\section{CONTEXT FOR RESEARCH}

Most of the data reported in this paper were collected from a sophomore-level quantum mechanics course taught at the UW by two different instructors during two different academic quarters. One class had an enrollment of 161; the other had 54 students. The course uses a spin-first approach that covers the first five chapters of McIntyre's Quantum Mechanics: A Paradigms Approach [7], supplemented by interactive lecture-tutorials based on Tutorials in Physics: Quantum Mechanics [8].

Supporting data from two junior-level quantum mechanics courses are also presented. Different instructors taught these courses, but both used the same textbook by Griffiths [9] and the tutorials [8]. There were 66 and 19 students enrolled in those two courses, respectively. All the students in the junior-level courses had previously completed the sophomore, spin-first course, but most (74 out of 85) had taken it prior to when we were conducting research in that course.

\section{OPEN-ENDED QUESTIONS}

Two sets of questions were given to probe student conceptual understanding of relative phases and their measurable effects. The first is in the context of spin; the second is based on the infinite square well potential. Each question was given as part of an online survey on which student responses were graded for completeness but not for correctness. 
Q1A. Consider two ensembles of spin-1/2 particles:

The particles in ensemble $A$ are all described by the state vector $\left|\psi_{A}\right\rangle=\frac{1}{2}|+\rangle_{Z}+\frac{\sqrt{3}}{2}|-\rangle_{z}$. The particles in ensemble B are all described by the state vector $\left|\psi_{B}\right\rangle=\frac{1}{2}|+\rangle_{z}+i \frac{\sqrt{3}}{2}|-\rangle_{z}$.

Is there an experiment that can be used to distinguish the particles in ensemble A from the particles in ensemble B? If your answer is yes, describe the experiment. If not, explain why not.

Q1B. State vectors are:

$\left|\psi_{A}\right\rangle=\frac{1}{2}|+\rangle_{Z}+\frac{\sqrt{3}}{2}|-\rangle_{Z}$ and $\left|\psi_{B}\right\rangle=\frac{1}{2}|+\rangle_{Z}-\frac{\sqrt{3}}{2}|-\rangle_{Z}$

Q1C. State vectors are:

$\left|\psi_{A}\right\rangle=\frac{1}{\sqrt{2}}|+\rangle_{Z}-\frac{1}{\sqrt{2}}|-\rangle_{Z}$ and $\left|\psi_{B}\right\rangle=\frac{1}{\sqrt{2}}|+\rangle_{Z}-i \frac{1}{\sqrt{2}}|-\rangle_{Z}$

FIG 1. Three versions of a question about relative phase in a spin context given in one sophomore- and two juniorlevel courses. (Versions $\mathrm{B}$ and $\mathrm{C}$ are identical to version $\mathrm{A}$ except that the state vectors have different coefficients.)

\section{A. Spin question}

Figure 1 shows three versions (Q1A, Q1B, and Q1C) of the spin question. Version Q1A was given to a sophomorelevel quantum course after lecture instruction on SternGerlach experiments and measurements. Versions Q1B and Q1C were given to two junior-level quantum courses after lecture instruction on spin.

In each question, students were told to consider two ensembles of spin-1/2 particles. The particles in each ensemble are represented by a superposition state written in the $S_{z}$ basis. The coefficients for each superposition state have identical magnitudes but different relative phases (e.g., the relative phases for $\left|\psi_{A}\right\rangle$ and $\left|\psi_{B}\right\rangle$ in Q1A are 0 and $\pi / 2$, respectively). We varied the coefficients in the different versions to assess whether any difficulties identified occur only in a particular case. All versions of this question asked students whether or not there is an experiment that can be used to distinguish the particles between the two ensembles. The structure of this question is very similar to the questions that we have previously used to investigate student ability to distinguish superposition states and mixed states [1].

Although this question does not require detailed computations, students need to know how to determine probabilities of different components of spin. For example, the probability of measuring $+\hbar / 2$ for $S_{x}$ can be found by taking the modulus square of the inner product between the eigenstate $|+\rangle_{x}$ and the state vector $|\psi\rangle:\left|{ }_{x}\langle+\mid \psi\rangle\right|^{2}$. Since the states are written in the $S_{z}$ basis, students need to know how to convert from the $S_{x}$ (or $S_{y}$ ) basis to the $S_{z}$ basis. For each ensemble, the probabilities of measuring either $+\hbar / 2$ or $-\hbar / 2$ for $S_{z}$ are identical since the magnitudes of the inner products are the same. However, the probabilities of Q2. Consider two ensembles of identical particles in infinite square well potentials. There is one particle in each well.

The particles in ensemble $\mathrm{C}$ are all described by the wave function $\psi_{C}(x)=\frac{1}{\sqrt{2}} \varphi_{1}(x)+\frac{1}{\sqrt{2}} \varphi_{2}(x)$.

The particles in ensemble $D$ are all described by the wave function $\psi_{D}(x)=\frac{1}{\sqrt{2}} \varphi_{1}(x)+i \frac{1}{\sqrt{2}} \varphi_{2}(x)$.

Is there an experiment that can be used to distinguish the particles in ensemble $C$ from the particles in ensemble $D$ ? If your answer is yes, describe the experiment. If not, explain why not.

FIG 2. A question about relative phase in the infinite square well context that was given in a sophomore-level course.

measuring either $+\hbar / 2$ or $-\hbar / 2$ for $S_{x}$ or $S_{y}$ are different since the inner products yield numbers with different magnitudes. Thus, one can distinguish the particles from different ensembles by measuring either the $x$ - or $y$-component of the spin.

\section{B. Infinite square well potential question}

An analogous question using the infinite square well context is shown in Fig. 2. It was administered after lecture instruction on the infinite square well potential in the sophomore course. The probability of measuring the ground state energy (and first excited state energy) is $1 / 2$ for the particles in each ensemble. However, the states have different position-space probability densities. By convention, the energy eigenfunctions for the infinite square well are taken to be real. The probability density for particles in ensemble $\mathrm{C}$ is $1 / 2\left(\varphi_{1}^{2}(x)+\varphi_{2}^{2}(x)+\right.$ $\left.2 \varphi_{1}(x) \varphi_{2}(x)\right)$ and the probability density for particles in ensemble $\mathrm{D}$ is $1 / 2\left(\varphi_{1}^{2}(x)+\varphi_{2}^{2}(x)\right)$. Hence, the probability density for the particles in ensemble $\mathrm{D}$ is symmetric about the midpoint of the well, whereas for ensemble $\mathrm{C}$ it is asymmetric, with higher probability on the left. Thus, one can distinguish the particles from those two ensembles by measuring position.

\section{RESULTS}

Student responses were analyzed by two researchers independently. After discussing and resolving disagreements, the inter-coder agreement is greater than $90 \%$. Table I shows student performance on all the questions. About two-thirds of the sophomore students correctly answered that particles from ensembles A and B are experimentally distinguishable. However, only about one-third described a correct experiment that can be used to distinguish them. Junior students in different quarters performed similarly, although Q1B has real coefficients and $\mathrm{Q} 1 \mathrm{C}$ has complex coefficients. In both quarters, only half of the students gave a correct answer and about one-third of the students gave correct reasoning. The common lines of 
Table I. Performance on questions about relative phase.

\begin{tabular}{l|c|c|c|c}
\hline \hline $\begin{array}{c}\text { Questions about } \\
\text { relative phase }\end{array}$ & $N$ & $\begin{array}{c}\text { Correct } \\
\text { answer } \\
\text { (yes) }\end{array}$ & $\begin{array}{c}\text { Correct } \\
\text { reasoning }\end{array}$ & $\begin{array}{c}\text { Incorrect } \\
\text { answer } \\
\text { (no) }\end{array}$ \\
\hline Q1A-Sophomore & 130 & $64 \%$ & $36 \%$ & $36 \%$ \\
\hline Q1B-Junior & 55 & $47 \%$ & $27 \%$ & $53 \%$ \\
\hline Q1C-Junior & 13 & $53 \%$ & $33 \%$ & $47 \%$ \\
\hline Q2-Sophomore & 126 & $47 \%$ & $17 \%$ & $53 \%$ \\
\hline \hline
\end{tabular}

reasoning are very similar for the two populations. Thus we discuss the responses from all students together.

About one-third of the sophomore students incorrectly stated that particles in ensembles $\mathrm{A}$ and $\mathrm{B}$ are indistinguishable. The most common incorrect reasoning, given by about $20 \%$ of the students, was that the probabilities are the same since a modulus square is used to determine probabilities. Many of these students did not specify whether they were referring to probabilities for the $z$-component alone or to probabilities for all the components. Less than $5 \%$ explicitly stated that the probabilities for measuring all three spin components $(x, y$, and $z$ ) of the spin are the same. Since the states are written in the $S_{z}$ basis, it is likely that some students did not invoke measurements of components other than the $z$-component.

Although uncommon (about 5\%), we found that some sophomores and juniors reasoned that imaginary numbers cannot be measured, an idea that has been previously discussed in the research literature [10]. For example, one student wrote: "There is no experiment to distinguish them, because the $i$ will go away when you measure it. Imaginary things cannot be measured." It is true that the probabilities and eigenvalues of the observables must be real, but imaginary coefficients are necessary to represent differences in how the basis states interfere with one another. This result suggests that these students do not understand the importance of the complex numbers.

Students performed less well on Q2 than on Q1A. About half of the students correctly answered that the ensembles are experimentally distinguishable and only about $17 \%$ gave correct reasoning. Few (less than 5\%) reasoned that the relative phase is different or that one can measure a different basis. Although these students did not describe a specific experiment, they did provide a correct explanation of the underlying physics and we categorized their responses as "correct reasoning." About 12\% described a correct experiment. The experiment they described involved measuring either position or momentum (which is also correct). Interestingly, some students (12\%) stated that the ensembles can be distinguished by measuring along a different direction. It may be that students recognized this question as similar to the one in the spin context but were not able to generalize the idea to a new context.

Many students (24\%) incorrectly reasoned that the probabilities are the same since the magnitudes of the
Q3. Consider a spin state $|\beta\rangle=\sqrt{\frac{3}{5}}|+\rangle_{z}+\sqrt{\frac{2}{5}}|-\rangle_{z}$.

A. Calculate the probability of measuring spin up in the $x$ direction.

B. If the state is replaced by $\left|\beta^{\prime}\right\rangle=\sqrt{\frac{3}{5}}|+\rangle_{z}+i \sqrt{\frac{2}{5}}|-\rangle_{z}$, will the probability be the same as or different from the probability you computed above?

FIG 3. A question probing student understanding of relevant formalism given to sophomore students.

coefficients are the same. This response is similar to the most common incorrect response on Q1. Most of these students did not specify whether they were referring to probabilities for energies alone or probabilities for all the observables. However, about $5 \%$ explicitly stated that the particles have the same probability density, which is consistent with prior research on student ability to use superposition for position-space wave functions with different relative phases [11].

We also found that very few students $(6 \%)$ used the term "phase" or "relative phase" among all responses. In addition, junior-level students were more likely to use the term "phase." The results suggest that students tend not to think spontaneously about the phases of complex numbers.

\section{STUDENT PERFORMANCE ON QUESTION WITH SPECIFIC PROMPT}

On questions like those described thus far, we found that many students did not recognize that two superposition states differing only by a relative phase are experimentally distinguishable. They often tended to overlook the importance of relative phases and complex numbers. It seemed that few invoked measurements of observables corresponding to bases other than what was given. We thus administered a question prompting students to compare probabilities for a specific observable to see whether performance would improve.

Figure 3 shows a question administered on a midterm exam in a different academic quarter of the sophomore course, after lecture instruction on Stern-Gerlach experiments, operators, and measurements. The question has two parts. Part A asks for the probability of measuring $+\hbar / 2$ for $S_{x}$ for a state written in the $S_{z}$ basis. In part B, students are asked to compare this probability to the probability of measuring the same quantity for a new state that differs only by a relative phase. Although this question does not explicitly ask for the probability in part B, we found that about three-quarters of the students attempted some calculations. Student performance on part B only is shown in Table II. We do not discuss student performance on part A separately since student performance on part B provides information about their performance on part A.

Although many students (about 69\%) correctly answered that the probabilities of measuring $+\hbar / 2$ for $S_{x}$ are 
Table II. Performance of sophomore-level students on a question with specific prompt.

\begin{tabular}{c|c|c|c}
\hline \hline $\begin{array}{c}\text { Q3- } \\
\text { Sophomore }\end{array}$ & $\begin{array}{c}\text { Correct } \\
\text { answer } \\
\text { (different) }\end{array}$ & $\begin{array}{c}\text { Correct } \\
\text { computation } \\
\text { /explanation }\end{array}$ & $\begin{array}{c}\text { Incorrect } \\
\text { answer } \\
\text { (same) }\end{array}$ \\
\hline$(N=52)$ & $69 \%$ & $46 \% *$ & $31 \%$ \\
\hline \hline
\end{tabular}

$* 40 \%$ correctly computed the probabilities; $6 \%$ explained that the probability amplitudes have different magnitudes.

different for the two states, only about two-fifths correctly determined the probability for the new state. The most common error $(13 \%)$ was treating a modulus square as "regular" square. Most of these students used vertical bars in their calculations (not parentheses), but then performed the computation as if they were squaring, with no complex conjugate. Lastly, they arrived at a complex probability represented by a complex number and concluded that the probabilities are different. A few students $(6 \%)$ argued that the probability amplitudes have different magnitudes without actually computing the probabilities.

About one-third of the students answered that the probabilities are the same. The most common incorrect response (13\%) was of the form $|a+i b|^{2}=|a+b|^{2}$. Some of those students appeared to neglect the cross term of $|a+b|^{2}$, while others reasoned that $|i|^{2}=1$. Another common error (9\%) was failing to change basis (including students who incorrectly changed basis by multiplying the $S_{z}$ basis by $1 / \sqrt{2}$ ).

Regardless of which answer they gave (that the probabilities are same or different), many students (27\%) had difficulty with the modulus square $|a+i b|^{2}$. They either treated it as a square, $(a+i b)^{2}$, or confused it with $|a+b|^{2}[11]$. About $17 \%$ of the students seemed to have difficulty with the formalism for determining probabilities. Those students made such errors consistently in both part A and part B.

\section{DISCUSSION}

The results in this study suggest that many students do not recognize the measurable effects of relative phases. For two superposition states that differ only by a relative phase, students often say that that the two states are experimentally indistinguishable since the magnitudes of the coefficients are the same. It seems that many students focus on the measurements of observables corresponding to bases that are given and do not invoke measurements of other observables. This occurs even if students have studied Stern-Gerlach experiments (including measurements on different components of spin) through both the textbook [7] and the course lectures. In particular, the course lectures and textbook in this study explicitly discussed how to use the experimental results to infer the coefficients for the expressions of the $S_{x}$ and $S_{y}$ basis states written in the $S_{z}$ basis. Thus, students have seen similar situations in which the relative phases affect probabilities. It seems that the errors they made are not simply due to a neglect of invoking measurements on different observables, but potentially reveals student perception of the usage and the role of complex numbers in quantum mechanics.

The difficulties not only occur when the coefficients are complex numbers, but also arise when the coefficients are purely real with opposite signs. We have observed similar problems in both spin and infinite square well contexts. Furthermore, students rarely used the term "relative phase" or even "phase". It seems that many students do not tend to think spontaneously about the phases of complex numbers. Thus, it is possible that even students who recognize that the states given in the questions are experimentally distinguishable are not able to generalize the idea that the relative phases could have measurable effects.

We have also found that many of the sophomore-level students not only lack an understanding of how relative phase can affect probabilities, but also have difficulty in determining probabilities. Some struggle with the quantum formalism that is related to determining probabilities. Others are not fluent with complex numbers - in particular how to determine the modulus square.

More research needs to be done in order to deepen our insights into student understanding of the role of complex numbers in quantum mechanics. It might be worthwhile to investigate whether or not student perceptions are affected by how complex numbers are used in classical mechanics, such as electrodynamics, a context in which complex numbers are primarily used to simplify computations.

\section{ACKNOWLEDGMENTS}

The authors acknowledge the valuable input from Sheh Lit Chang to this study. We would also like to thank UW faculty members for welcoming this research in their courses. This study is funded through NSF grant 1022449 .
[1] G. Passante, P. J. Emigh, \& P. S. Shaffer, Phys. Rev. ST Phys. Educ. Res., 11, 020135 (2015).

[2] G. Zhu \& C. Singh, Am. J. Phys. 80 (3), 252-259 (2012).

[3] H. G. Close et al., PER Conf. Proc. (2013).

[4] E. M. Smith et al., PER Conf. Proc. (2015).

[5] T. Wan et al., PER Conf. Proc. (2016).

[6] P. J. Emigh, G. Passante, and P. S. Shaffer, Phys. Rev. ST Phys. Educ. Res. 11, 020112 (2015).
[7] D. H. McIntyre, Quantum Mechanics: A Paradigm Approach, $1^{\text {st }}$ edition (2012).

[8] L. C. McDermott et al., Tutorials in Physics: Quantum Mechanics, Preliminary edition (2016).

[9] D. J. Griffiths, Introduction to Quantum Mechanics, 2nd edition, Prentice Hall (2005).

[10] G. Passante, PER Conf. Proc. (2016).

[11] P. J. Emigh et al., PER Conf. Proc. (2016). 